\title{
WASHINGTON BEAT
}

\section{Challenges in Laboratory Coding 2015}

\section{KATHLEEN HANSEN}

\begin{abstract}
ABBREVIATIONS: CLFS - Clinical Laboratory Fee Schedule, CPT - Current Procedural Terminology, AMA - American Medical Association, CMS - Center for Medicare and Medicaid Services, HCPCS Healthcare Common Procedure Coding System, NGS Next Generation Sequencing, MAC - Medicare Administrative Contractor, Mopath - molecular pathology, MUE - Medically Unlikely Edit
\end{abstract}

INDEX TERMS: CPT coding, molecular pathology, drug screening, immunohistochemical stains

\section{Clin Lab Sci 2015;28(1):3}

Katbleen Hansen, MLS(ASCP) ${ }^{C M}$, Advisor, ASCLS Government Affairs Committee, Laboratory Administration Consultant, Fairview Health Services, Minneapolis, $M N$

Address for Correspondence: Katbleen Hansen, MLS(ASCP) ${ }^{C M}, 2309$ Beverly Road, St. Paul, MN 55104,651-983-8613 khansen3@fairview.org

\section{INTRODUCTION}

Laboratory managers and others have long been familiar with the use of Current Procedural Terminology (CPT) codes to identify services provided by the laboratory for billing and reporting purposes. The CPT manual is the property of the American Medical Association (AMA) and is updated annually, including the laboratory section, which encompasses CPT codes 80047-89398.

Until recently, the typical year's update consisted of 1015 new or modified codes. Updates are announced in early summer, and the Center for Medicare and Medicaid Services (CMS) holds a mid-July meeting each year at which stakeholders present testimony about the new codes and make suggestions as to how they should be priced by CMS. ASCLS Executive Vice President Elissa Passiment attends this meeting to present our views, or alternatively ASCLS submits testimony in writing. Final codes and instructions for their use are published in the fall.

Reimbursement amounts are published near the end of the year with the new Clinical Laboratory Fee Schedule (CLFS). Reimbursement amounts for the CLFS are determined using one of two methodologies: crosswalk or gap fill. Crosswalk matches the new code with an existing code that is deemed to be of similar cost to perform, and assigns that same reimbursement to the new code. Gap fill assumes that the new code is sufficiently different from existing codes to need its own reimbursement determination. Each Medicare contractor decides what its reimbursement will be based on its determination of the cost to perform the test. These different amounts are used for a year, after which Medicare reviews and assigns an amount on the CLFS. The laboratory community has traditionally been more comfortable with the crosswalk method since the gap fill method can lead to very different reimbursements in different contractor jurisdictions, and amounts may not reflect the laboratory community's assessment of cost to perform the test.

For Medicare, CMS usually follows what the AMA publishes, but sometimes chooses to establish Healthcare Common Procedure Coding System (HCPCS) codes instead. This often requires the laboratory to bill CMS (and sometimes Medicaid as well) using a HCPCS code and bill private payers using the AMA CPT code.

The last three years, significantly more new laboratory codes have been introduced than in past years. One hundred or more new codes have been introduced for 2015, along with complex "roll-up" or substitution rules which present a challenge to laboratory management, coders, and information systems.

The purpose of this paper is to raise awareness and understanding of the implications of recent and near future laboratory coding changes and the challenges that these present. 


\section{WASHINGTON BEAT}

\section{Molecular pathology coding}

Prior to January 1, 2013 "stacking" codes 83890-83914 were used to describe all molecular tests using codes that described the steps in the assay such as extraction, purification, probes, sequencing, interpretation and others. Multiple units of codes, such as probes, were allowed. This system led to laboratories coding differently for the same gene assay and did not allow the payers to know the gene analyzed. In the $2012 \mathrm{CPT}$ manual, the AMA introduced a new set of gene-based codes for molecular testing. Payers, including CMS, did not adopt the new codes for 2012, but they were required beginning January 1, 2013, when the stacking codes were eliminated from the CPT manual.

- Each Tier 1 code (81200-81355) is assigned to a specific gene or group of genes and may only be used for that gene(s). (109 codes)

- Tier 2 codes 81400-81408 (Levels 1-9) describe methodology and complexity. Each of the nine Tier 2 codes has approximately 40-80 genes listed in the CPT manual that fit the category. The number of examples nearly doubled in 2014 with the addition of over 300 more genes names to the Tier 2 codes. A Tier 2 code may not be used unless the specific gene being tested appears in the list for that CPT code. This rule is often misunderstood by performing laboratories as they may consider the genes listed to be examples rather than the entire list included.

- $\quad$ CPT code 81479 "Unlisted molecular pathology procedure" is used for all other molecular tests. Hundreds, if not thousands, of genes fall into this category.

\section{Challenges with Mopath coding}

None of the 2014 Tier 2 codes describes Next Generation Sequencing (NGS), a fast growing technique. This leads to a coding dilemma. Is it best to use 81479 for all NGS testing? This is not a desirable option since many payers do not pay 81479 . Or is it better to choose the specific gene name from under a Tier 2 codes that describes sequencing? The longstanding rule that an analyte should be coded to the greatest specificity seems to favor the latter approach.

Payment for Mopath is inconsistent. Some Medicare Administrative Contractors (MAC's) have restrictive Local Coverage Decision (LCD) policies that list the codes they will reimburse. Many don't pay for 81479 at all. Many MACs currently are revising LCDs. Look at the web site for your MAC to see whether a draft LCD is posted for comment.

At least one Medicare contractor, National Government Services has an LCD for Region J6 that requires the specific gene name to appear on the claim for Tier 2 codes and 81479 . Many billing systems are set up to only send the CPT and the price, not a description.

The 2015 CPT manual will include new Tier 1 codes for three genes: FLT3, MLH1, and PCA3/KLK311. Twenty-one new codes are listed for panels of genes, most performed by next generation sequencing. Complete code numbers had not been released at the time of writing but they will be in the $814 \mathrm{xx}$ range. Examples include:

- 814XX Hearing loss (eg, nonsyndromic hearing loss, Usher syndrome, Pendred syndrome); duplication/deletion analysis panel, must include copy number analyses for STRC and DFNB1 deletions in GJB2 and GJB6 genes

- 814XX Hereditary colon cancer syndromes (eg, Lynch syndrome, familial adenomatosis polyposis); genomic sequence analysis panel, must include analysis of at least 7 genes, including APC, CHEK2, MLH1, MSH2, MSH6, MUTYH, and PMS2

The remainder of the new 2015 codes can be found at http://www.cms.gov/Medicare/Medicare-Fee-for-Servic e-Payment/ClinicalLabFeeSched/Laboratory_Public_M eetings.html. Click on the PDF of Clinical Laboratory Fee Schedule CY 2015 Updates.

\section{Immunohistochemical stains}

In the 2014 CPT manual, the AMA changed the description of CPT 88342 and added a new code 88343 as follows:

- 88342 Immunohistochemistry or immunocytochemistry, each separately identifiable antibody per block, cytologic preparation, or hematologic smear; first separately identifiable antibody per slide (formerly 88342 was defined as per antibody)

- 88343 each additional separately identifiable antibody per slide (list in addition to code for primary procedure)

Note: 88343 is used only if more than one antibody is 


\section{WASHINGTON BEAT}

evaluated on the same slide (as with multiplex stains).

CMS disagreed with the AMA descriptions and created new HCPCS codes:

- G0461 Immunohistochemistry or immunocytochemistry, per specimen; first single or multiplex antibody stain

- G0462 Immunohistochemistry or immunocytochemistry, per specimen; each additional single or multiplex antibody stain (list separately in addition to code for primary procedure)

It is common to have multiple specimens on a surgical case, such as bone marrow biopsy and lymph nodes; breast tissue and lymph nodes, and many other examples. A specimen is defined as tissue that is submitted, identified, and reported separately.

A great deal of confusion resulted about the use of these HCPCS codes. Many coders and payers have interpreted that a one-to-one correspondence exists between 88342 and G0461 and between 88343 and G0462. This is not true. Instead, a laboratory should code one G0461 for the first stain on each specimen and multiple G0462's corresponding to the number of additional stains on the same specimen.

Payers are not consistent in their claim requirements for modifiers and rolling up quantities of the same CPT. A modifier 59 "Distinct procedural service" may be used to communicate that multiples of the same CPT code are not an erroneous duplication. Valid multiples of the same CPT code many be rolled up to one line on a claim with a number indicating the quantity being billed. Consult the CPT manual for more information about modifiers and your billing office for roll-up requirements.

ASCLS has commented on proposed Medically Unlikely Edits (MUEs) for G0461 and G0462, which seem to be adequate for the numbers of specimens and stains in most surgical cases. MUE's limit the number of a given CPT code that will be paid on a given date of service.

Many laboratories face the work flow issue of how to determine how many specimens are in a given case and convert to the appropriate number of HCPCS codes, which is not easily automated. Reimbursement for
G0461 and G0462 is significantly lower than for 88342, especially for the professional fees.

\section{Coding for drug testing}

The CPT manual for 2015 also includes major changes for both qualitative and quantitative drug tests. Many old CPT's are being deleted and replaced.

\section{Screening tests}

Drug screen codes 80100, 80101, 80102, 80103, and 80104 are deleted and replaced with the following (italics added by this author)

- 803XX: Drug screen, any number of drug classes from Drug Class List A; any number of nonTLC devices or procedures, (eg, immunoassay) capable of being read by direct optical observation including instrumented-assisted when performed (eg, dipsticks, cups, cards, cartridges), per date of service (similar to 80104)

- 803XX: Drug screen, any number of drug classes from Drug Class List A; single drug class method, by instrumented test systems (eg, discrete multichannel chemistry analyzers utilizing immunoassay or enzyme assay), per date of service (similar to 80101)

- 803XX: Drug screen, presumptive, single drug class from Drug Class List B, by immunoassay (eg, ELISA) or non-TLC chromatography without mass spectrometry (eg, GC, HPLC), each procedure

- 803XX: Drug screen, any number of drug classes, presumptive, single or multiple drug class method; thin layer chromatography procedure(s) (TLC)(eg, acid, neutral, alkaloid plate), per date of service

- 803XX: Drug screen, any number of drug classes, presumptive, single or multiple drug class method; not otherwise specified presumptive procedure (eg, TOF, MALDI, LDTD, DESI, DART), each procedure (similar to 80100)

In recent years, CMS has required the use of HCPCS code G0431 and G0434 in place of 80100 and 80101, which are among the $80 \mathrm{XXX}$ codes being eliminated. It is unclear at this time whether CMS will use the new 803XX screening codes listed above or continue to require the use of G0431 and G0434. (See Table 1)

VOL 28, NO 1 WINTER 2015 CLINICAL LABORATORY SCIENCE 
Table 1. Drug Classes A and B for 2015 CPT coding

\begin{tabular}{ll}
\hline \multicolumn{1}{c}{ Drug Class A } & \multicolumn{1}{c}{ Drug Class B } \\
\hline Alcohol (Ethanol) & Acetaminophen \\
Amphetamines & Carisoprodol/Meprobamate \\
Barbiturates & Ethyl Glucuronide \\
Benzodiazepines & Fentanyl \\
Buprenorphine & Ketamine \\
Cocaine metabolite & Meperidine \\
Heroin metabolite & Methylphenidate \\
Methadone & Nicotine/Cotinine \\
Methadone metabolite (EDDP) & Salicylate \\
Methamphetamine & Synthetic Cannabinoids \\
Methaqualone & Tapentadol \\
Methylenedioxymethamphetamine & Tramadol \\
Opiates & Zolpidem \\
Oxycodone & Drug not otherwise specified \\
Phencyclidine & \\
Propoxyphene & \\
Tetrahydrocannabinol metabolites & \\
Tricyclic Antidepressants & \\
\hline
\end{tabular}

\section{Quantitative tests}

Most CPT codes in the Therapeutic Drug Assays section remain (80150-80299). A few are deleted (80152, 80154, 80160, 80166, 80172, 80174, 80182, 80196), revised $(80162,80164,80171,80299)$, or added (801xx Free Digoxin, 801XX Free Valproic Acid).
All drug CPT codes that were embedded in the Chemistry section (82XXX-84XXX) are deleted.

In addition, new coding/bundling rules have been added for billing for drugs, according to how many individual drugs in that classification are measured.

The remainder of the changes in drug codes can be found at the web site listed earlier. Actual CPT codes and guidance on their use will be available in late August 2014.

It is not clear how to code drugs that have their own code in the 80150-80299 series but also appear in one of the bundling groups? For example if phenytoin is ordered singly, does one use the 80185 , or the bundling code for 1-2 anti-epileptics?

Laboratories will require assistance from the IT department to determine how to operationalize the bundling rules, whether in the LIS or the billing system. Note in Table 2 that different drug classes have different groupings for bundling.

Table 2. Examples of roll-up codes for quantitative drug assays:

\begin{tabular}{|c|c|c|c|}
\hline $\begin{array}{l}\text { Placeholder } \\
\text { CPT code }\end{array}$ & Drug Class & Number of drugs & Drugs included in this category \\
\hline $803 X X$ & Antidepressants, serotonergic class & $1-2$ & $\begin{array}{l}\text { Citalopram, duloxetine, escitalopram, } \\
\text { fluoxetine, fluvoxamine, paroxetine, sertraline }\end{array}$ \\
\hline 803XX & & $3-5$ & \\
\hline $803 X X$ & & 6 or more & \\
\hline $803 X X$ & $\begin{array}{l}\text { Antidepressants, tricyclic and } \\
\text { other cyclicals }\end{array}$ & $1-2$ & $\begin{array}{l}\text { Amitriptyline, amoxapine, clomipramine, } \\
\text { demexiptiline, desipramine, doxepin, imipramine, } \\
\text { maprotiline, mirtazpine, nortriptyline, } \\
\text { protriptyline, }\end{array}$ \\
\hline $803 X X$ & & $3-5$ & \\
\hline $803 X X$ & & 6 or more & \\
\hline $803 X X$ & $\begin{array}{l}\text { Antidepressants, not otherwise } \\
\text { specified }\end{array}$ & each & $\begin{array}{l}\text { Bupropion, desyenlafaxine, isocarboxazid, } \\
\text { nefazodone, phenelzine, selegiline, } \\
\text { tranylcypromine, trazodone, venlafaxine }\end{array}$ \\
\hline $803 X X$ & $\begin{array}{l}\text { Anti-epileptics, not otherwise } \\
\text { specified }\end{array}$ & $1-3$ & $\begin{array}{l}\text { Carbamazepine, clobazam, diamethadione, } \\
\text { ethosuximide, ezogabine, lamotrigine, } \\
\text { levetiracetam, methsuximide, oxcarbazepine, } \\
\text { phenytoin, primidone, rufinamide, tiagabine, } \\
\text { topiramate, trimethadione, valproic acid, } \\
\text { zonisamide }\end{array}$ \\
\hline $803 X X$ & & $4-6$ & \\
\hline $803 X X$ & & 7 or more & \\
\hline
\end{tabular}


WASHINGTON BEAT

\begin{tabular}{llll}
\hline $\begin{array}{l}\text { Placeholder } \\
\text { CPT code }\end{array}$ & \multicolumn{1}{c}{ Drug Class } & Number of drugs & \multicolumn{1}{c}{ Drugs included in this category } \\
\hline $803 \mathrm{XX}$ & $\begin{array}{l}\text { Antispychotics, not otherwise } \\
\text { specified }\end{array}$ & $1-3$ & $\begin{array}{l}\text { Aripiprazole, chlorpromazine, clozapine, } \\
\text { fluphenazine, haloperidol, loxapine, mesoridazine, } \\
\text { molindone, olanzapine, paliperidone, } \\
\text { perphenazine, phenothiazine, pimozide, } \\
\text { prochlorperazine, quetiapine, risperidone, } \\
\text { trifluoperazine, thiothixene, thoridazine, } \\
\text { ziprasidone }\end{array}$ \\
$803 \mathrm{XX}$ & & \\
$803 \mathrm{XX}$ & $4-6$ & 7 or more & \\
\hline
\end{tabular}

\section{Summary}

Many laboratory CPT changes exist for 2015 and many will require changes to information systems or manual review of claims. Laboratories are encouraged to begin work as soon as possible in order to avoid denied claims!

\section{REFERENCES}

1. Current Procedural Terminology CPT 2014, American Medical Association, Pathology and Laboratory, pp. 433-516
2. Current Procedural Terminology CPT 2015, American Medical Association, Pathology and Laboratory

3. http://www.cms.gov/Medicare/Medicare-Fee-for-ServicePayment/ClinicalLabFeeSched/Laboratory_Public_Meetings. html. Clinical Laboratory Fee Schedule CY 2015 Updates.

4. Medicare Claims Processing Manual, Centers for Medicare \& Medicaid Services , Publication \#: 100-04, Chapter 16, Laboratory Services., http://www.cms.gov/Regulations-andGuidance/Guidance/Manuals/Internet-Only-Manuals-IOMsItems/CMS018912.html 